\title{
Adapting computational optimization concepts from aeronautics to nuclear fusion reactor design
}

\author{
W. Dekeyser ${ }^{1, a}$, D. Reiter ${ }^{2}$, and M. Baelmans ${ }^{1}$ \\ ${ }^{1}$ KU Leuven, Department of Mechanical Engineering, Celestijnenlaan 300A, 3001 Leuven, Belgium \\ ${ }^{2}$ Institute of Energy and Climate Research, Forschungszentrum Juelich GmbH, EURATOM \\ Association, Trilateral Euregio Cluster, D-52425 Juelich, Germany
}

\begin{abstract}
Even on the most powerful supercomputers available today, computational nuclear fusion reactor divertor design is extremely CPU demanding, not least due to the large number of design variables and the hybrid micro-macro character of the flows. Therefore, automated design methods based on optimization can greatly assist current reactor design studies. Over the past decades, "adjoint methods" for shape optimization have proven their virtue in the field of aerodynamics. Applications include drag reduction for wing and wing-body configurations. Here we demonstrate that also for divertor design, these optimization methods have a large potential. Specifically, we apply the continuous adjoint method to the optimization of the divertor geometry in a 2D poloidal cross section of an axisymmetric tokamak device (as, e.g., JET and ITER), using a simplified model for the plasma edge. The design objective is to spread the target material heat load as much as possible by controlling the shape of the divertor, while maintaining the full helium ash removal capabilities of the vacuum pumping system.
\end{abstract}

\section{Introduction}

Due to the anisotropic transport in nuclear fusion reactors based on magnetic confinement, a large fraction of the heating power is deposited on a very small part of the confinement vessel, on rather narrow bands in the so-called divertor. This leads to strongly peaked power deposition profiles, with peaks often exceeding materials limits. Thus, the divertor is prone to severe material migration (erosion, deposition) and radiation damage. For the successful development of future fusion power plants, it is essential that divertors can be designed to adequately handle these large power and particle fluxes during steady state operation.

Presently, divertor design is heavily assisted by numerical simulation tools such as the current ITER divertor design code B2-EIRENE [1,2]. Typically, these codes are used in a design-by-analysis approach, where divertor configurations are manually adjusted to meet design requirements. Due to the complex nature of the flows, and the large number of design variables, however, this method is computationally extremely demanding.

In this paper, we address the divertor design problem from a different perspective, by using a shape optimization framework. In the next Section, we motivate the formulation of this novel

\footnotetext{
a e-mail : Wouter.Dekeyser@mech.kuleuven.be
}

This is an Open Access article distributed under the terms of the Creative Commons Attribution License 2.0, which permits unrestricted use, distribution, and reproduction in any medium, provided the original work is properly cited. 
approach, and point out how methods developed for aerodynamics can be used in edge plasma applications. Then, in Section 3 the method is applied to a model problem where the aim is to design targets for optimal power load spreading under ITER-relevant operating conditions. Rather than using a most complete model as implemented in B2-Eirene, we assess this divertor design strategy with a set of somewhat simplified governing 2D partial differential equations, which, however, captures most essential ingredients and nonlinear challenges of current divertor design codes. In Section 4, the results are discussed. Finally, conclusions and an outlook to further work are given in Section 5 .

\section{The optimization approach to divertor target design}

In general, the different design requirements and constraints, combined with the large number of control variables turn divertor design into a challenging problem. In the design process, the divertor shape, the magnetic configuration as well as transonic driven plasma and gas flows can be used in order to cope with the challenges and to achieve the desired performance. With a detailed parameterization of the divertor shape, and the use of several divertor coils, the number of control variables can easily exceed hundreds or even thousands. Furthermore, there may be constraints limiting the available design space related to for example the need to shield critical components from neutron bombardment. Last but not least, the physics governing the plasma edge is very complex, with ions and electrons interacting with neutrals in the vicinity of the vessel wall. Ions and electrons are typically modelled using (Navier-Stokes-like) fluid equations for mass, momentum and energy conservation, while neutrals require a kinetic treatment using Monte Carlo codes. Collision processes between the different particles (e.g. ionization, recombination, charge-exchange) then lead to source or sink terms in the different fluid equations. Numerical simulation of the plasma edge using codes such as B2-Eirene is therefore computationally very demanding. Presently, these codes are indispensable for the design of future divertor concepts, as they allow for the extrapolation of the current understanding of the edge plasma physics to fusion reactor relevant operating regimes. Due to the long simulation times, however, it is impossible to consider many different geometries and operating points during the design process.

Similar design challenges are often faced in aerodynamics. There, the aim may be to design air foils for drag reduction or lift maximization $[3,4]$, increase the pressure gain in compressor stages, or to shape ducts for minimum pressure losses [5]. In these applications, the flows are constrained by the Navier-Stokes equations. In the past years, these problems have been treated very effectively by using optimization approaches where the shape is considered as the design or control variable. The design objective is formulated as a cost functional, which is to be minimized by changing the shape of the air foil or duct. The Navier-Stokes equations are introduced as a constraint on the flow field. Using optimization algorithms, the best design for a specific design objective is automatically obtained, while ensuring that the model equations are satisfied at the optimum design point.

An essential feature of shape optimization problems constrained by partial differential equations is the huge cost associated with sensitivity analysis if straightforward finite difference approaches are used. Indeed, after discretization or parameterization of the shape, a large number of design variables results. Computing the gradient of the cost functional at a certain design point using finite differences requires perturbing each design variable in turn, and solving the governing model equations to determine its influence on the cost functional. This cost quickly becomes prohibitive. An adjoint or dual set of PDEs can be derived, however, which contains the necessary information on how to change all design variables in order to obtain a reduction in the cost functional. The adjoint problem typically has similar structure as the forward analysis problem, but has reversed characteristics. It can be solved at a comparable computational cost. Thus, independent of the number of design variables, the gradient of the cost functional with respect to all design variables can be computed at an equivalent cost of approximately two flow simulations, i.e. one solution of the forward system and one of the adjoint system. This gradient can then be used in traditional gradient descent algorithms, for example. Alternatively, the forward model equations, the adjoint equations, 
and the equation stating that the gradient of the cost functional must be zero at the optimum may be regarded as one large system of coupled (partial differential) equations, and solved simultaneously. Such methods are often called one-shot methods. Several authors have effectively used these methods in the field of aerodynamics design, see e.g. [6,7]. Using these methods, the complete shape optimization problem can be solved at an equivalent cost of roughly 5 to 10 forward flow simulations. Motivated by the success of the one-shot methods, we also apply them for the present study.

Apart from the automated design and the efficient solution algorithms using advanced adjoint methods, working in an optimization framework offers another important advantage. The optimization environment naturally allows for the incorporation of constraints. For example, in the design of the divertor, sufficient space is needed for neutron shielding. By imposing constraints on the design space, the optimizer can be forced to consider only those designs which allow for this shielding.

\section{An ITER-like model problem}

\subsection{Optimizing targets for power load spreading}

In this section, we address the issue of the peaked power deposition profile along the divertor targets through a shape optimization approach. In order to gain insight into how much this power peak can be reduced and how well the power can be spread out by controlling only the geometry of the divertor, we define the ideal target power load as a constant value $Q_{\mathrm{d}}$. A power load of $10 \mathrm{MW} \mathrm{m}^{-2}$ is generally considered as an upper limit in divertor design in order to avoid excessive material degradation, see e.g. [2]. Therefore, we take a flux density of $Q_{\mathrm{d}}=2.5 \mathrm{MW} \mathrm{m}^{-2}$ as our design objective, and we aim to minimize the square of the difference between $Q_{\mathrm{d}}$ and the actual target power flux density $Q$ integrated across the target surface. $Q$ contains both the heat transported to the target by plasma convection and conduction, as well as the deposited recombination energy as ions and electrons recombine at the target surface.

\subsection{Geometry}

In order to reduce complexity, we approximate the Scrape-Off Layer (SOL) as a thin cylindrical shell, in which the poloidal projections of the magnetic field lines are perfectly vertical, see Figure 1. Toroidal symmetry, as well as up-down symmetry is assumed. The dimensions are representative of a typical ITER plasma equilibrium. The length of the core region (EF) is $20 \mathrm{~m}$, and the length of the private flux walls (AE and FD) is $1 \mathrm{~m}$. In the radial direction, the shell has a constant width of $0.1 \mathrm{~m}$. The pitch of the magnetic field is taken constant at 0.1 . Note that this configuration does not take any magnetic effects such as flux tube expansion into account. As a starting point for the optimization, we have straight targets $(\mathrm{AB}$ and $\mathrm{CD})$. The final shape of the targets is to be determined by the optimizer. 

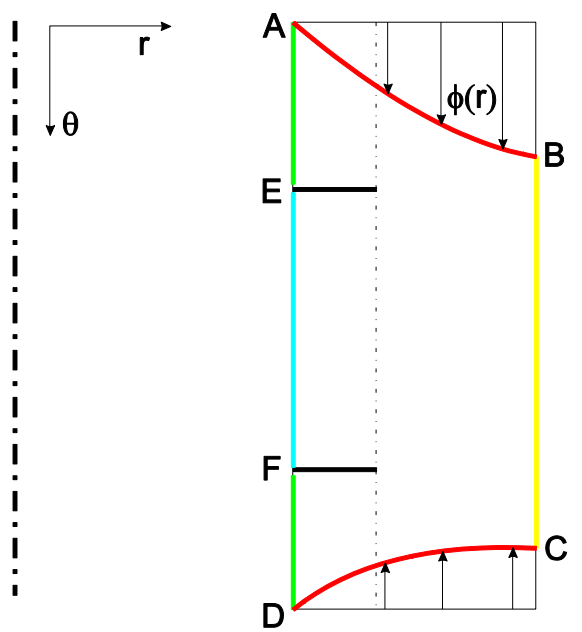

Fig. 1. Simplified cylindrical shell model for the SOL. Toroidal symmetry is assumed.

\subsection{Edge plasma model}

For initial design applications, it is desirable to have a physical model which describes the relevant physical processes with sufficient accuracy, yet is fast enough to allow for quick computations. The model for the edge plasma used in the present study is based on the models used in edge codes such as B2-Eirene, but makes additional simplifying assumptions. A single species Deuterium plasma is considered. The plasma density and velocity parallel to the magnetic field are governed by NavierStokes like continuity and momentum equations. In contrast to more complete edge models, it is assumed here that ions and electrons have the same temperature, and thus a single energy equation can be solved. Compared to previous work [8], where only conductive heat transport was considered, the energy equation has been significantly improved, however. Now also heat convection is included in the model, as well as source terms due to the conversion from kinetic to internal energy, leading to fully consistent parallel momentum and internal energy equations. Plasma transport coefficients in the direction parallel to the magnetic field are classical following Braginskii [9]. In the radial direction, anomalous transport coefficients are used to describe the dominant turbulent transport.

As a further simplification compared to full edge codes, the neutral component is not modelled by using a kinetic Monte Carlo code (such as Eirene), but rather neutral density is obtained through an isotropic neutral pressure diffusion equation. This leads to significant savings in terms of computational time. Neutrals are assumed to be in thermal equilibrium with ions (and thus also with electrons in this model). There is an isotropic contribution from the neutrals to the heat conduction coefficients.

The interactions between ions, electrons and neutrals are taken into account through analytical source terms in the model equations. Rate coefficients for electron impact ionization [10], radiative recombination [10] and charge-exchange [11] are simple functions of temperature. Although there are no separate balance equations for impurity ions, an artificial impurity radiation function takes the effect of non-equilibrium radiation cooling into account [12]. The impurity density is assumed to be a spatially constant fraction of the Deuterium density. The energy sink due to impurity radiation combined with the plasma momentum loss at charge-exchange collisions supply the necessary ingredients for (partial) detachment, an interesting operating regime with low divertor target temperatures and particle fluxes. 


\subsection{Model parameters and boundary conditions}

The parameters for the model problem are representative for a typical ITER-design case. There is $100 \mathrm{MW}$ input power from the core, and the ion density at the core interface is fixed at $5 \cdot 10^{19} \mathrm{~m}^{-3}$. At the targets, sheath conditions are applied, while at the wall and private flux boundaries we have radial decay lengths of $0.01 \mathrm{~m}$ for ion density and $0.10 \mathrm{~m}$ for the temperature. At all boundaries in contact with the vessel, there is a recycling coefficient of 1 . The thermal $\mathrm{D}_{2}$ pumping speed at the private flux is set to $30 \mathrm{~m}^{3} \mathrm{~s}^{-1}$. Values for the anomalous ion diffusivity and the anomalous ion and electron conductivities are all set to $1 \mathrm{~m}^{2} \mathrm{~s}^{-1}$. In the test case, we consider Carbon as an impurity. The relative Carbon concentration is chosen in such way to have approximately half of the SOL power removed by radiation in the initial configuration. This relative concentration, 0.0075 , is then kept constant during the optimization process.

\section{Results and discussion}

The model problem described above is discretized on a grid with 160 cells in the poloidal $(\theta)$ direction and 60 cells in the radial $(r)$ direction, and solved using a finite volume solver. Due to the highly nonlinear interactions between neutrals and plasma, reducing the residuals of the forward problem by three orders of magnitude requires approximately 100.000 outer iterations of the state equations using a time step of $10^{-6} \mathrm{~s}$. A similar reduction of the residuals during the optimization run, in which forward equations and adjoint equations are solved simultaneously using a one-shot approach, requires around 500.000 outer iterations of the coupled forward-adjoint system, while updating the grid at every iteration. As the latter system is twice as large as the system of forward equations alone, this means that the optimizer converges in a computational time only a factor 10 larger than the forward problem. It should be noted, however, that the plasma moves from an attached to a partially detached state during the optimization process (see discussion below). This physical transition in its own strongly reduces the convergence speed of the forward equations, and thus the factor of 10 in convergence speed reduction is only in part due to the optimizer.

Figure 2 shows the initial target configuration and power deposition profiles (blue), the desired constant load $Q_{\mathrm{d}}$ (red) and the optimized configuration (green). As expected, the anisotropy in transport properties does not allow for a perfectly homogeneous power profile, but the peak load is significantly reduced. Note that the peak of the power deposition profile has also broadened somewhat, and is more symmetric than in the initial configuration. The optimizer has taken full advantage of the presence of the neutrals to enhance the radial transport of energy. Looking at Figure 3, we see indeed that the divertor geometry has a large influence on the spatial distribution of the neutral component. In the initial configuration, neutral density peaked in the lower left corner of the private flux region. After optimization, the neutral cloud has moved to the separatrix area. As we discuss below, this leads to important changes in the energy balance. 

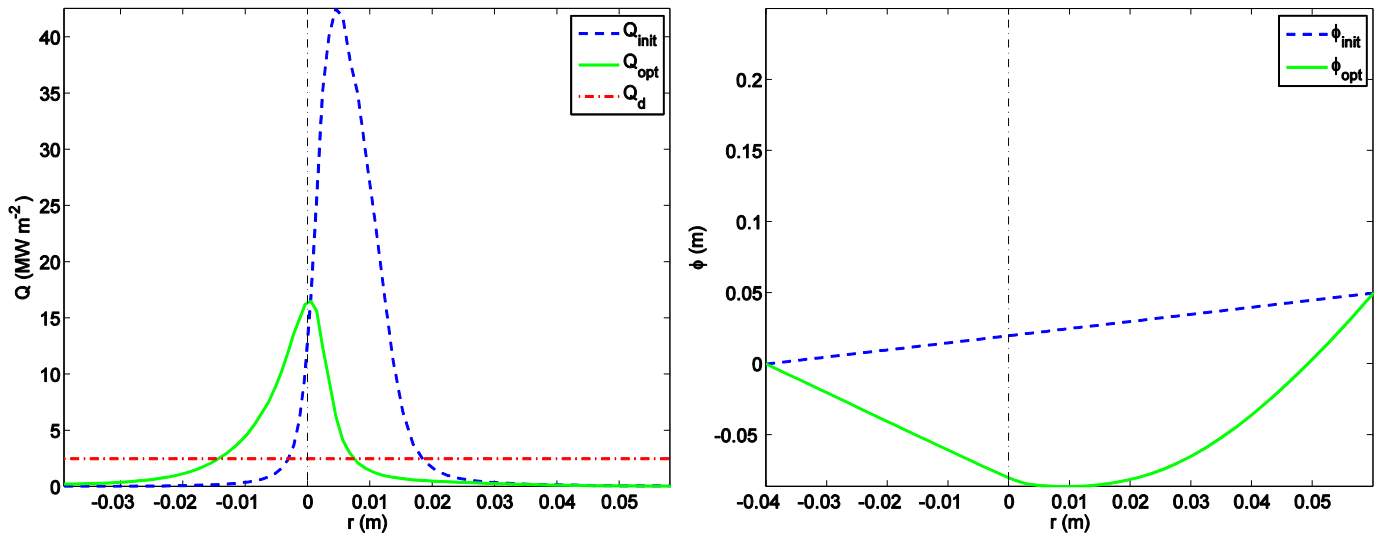

Fig. 2. Left: profile of power density across divertor target in initial configuration (blue), desired profile of $2.5 \mathrm{MW} \mathrm{m}^{-2}$ (red) and optimized profile (green). Right: the initial and optimized target geometries.
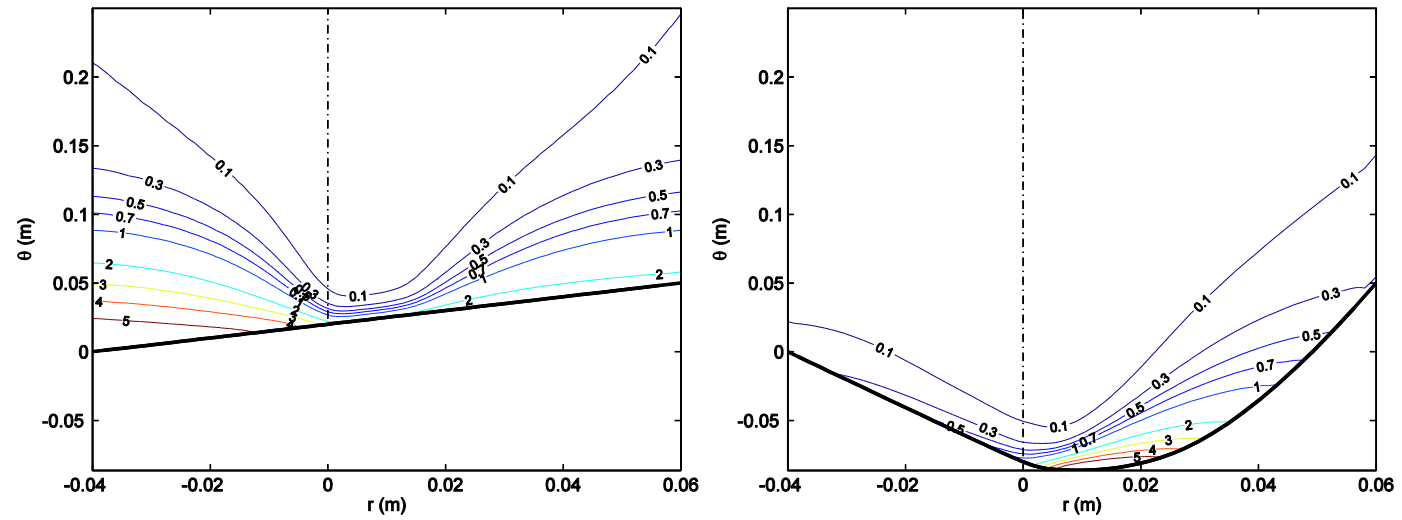

Fig. 3. Contours of neutral density in initial (left) and optimized (right) configurations $\left(10^{20} \mathrm{~m}^{-3}\right)$.

It is clear that the total amount of energy deposited on the targets due to convection, conduction and surface recombination is reduced compared to the initial configuration. The difference is mainly accommodated by increased radiation by impurities and increased dissipation during the ionization process through radiation from excited states. The radiation patterns of initial and optimized configurations are compared in Figures 4 and 5, respectively. Due to the isotropic nature of radiation, this power is distributed more homogeneously across the divertor surface. 

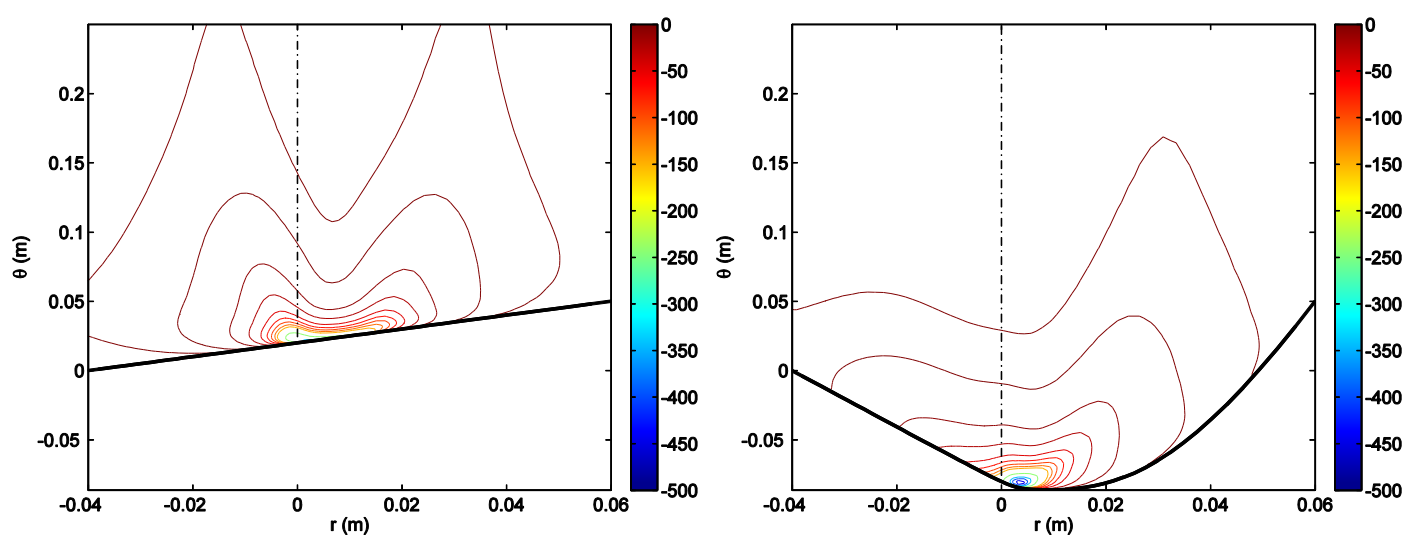

Fig. 4. Ionization energy losses in initial (left) and optimized (right) configurations, ( $\mathrm{MW} \mathrm{m}^{-3}$ ).
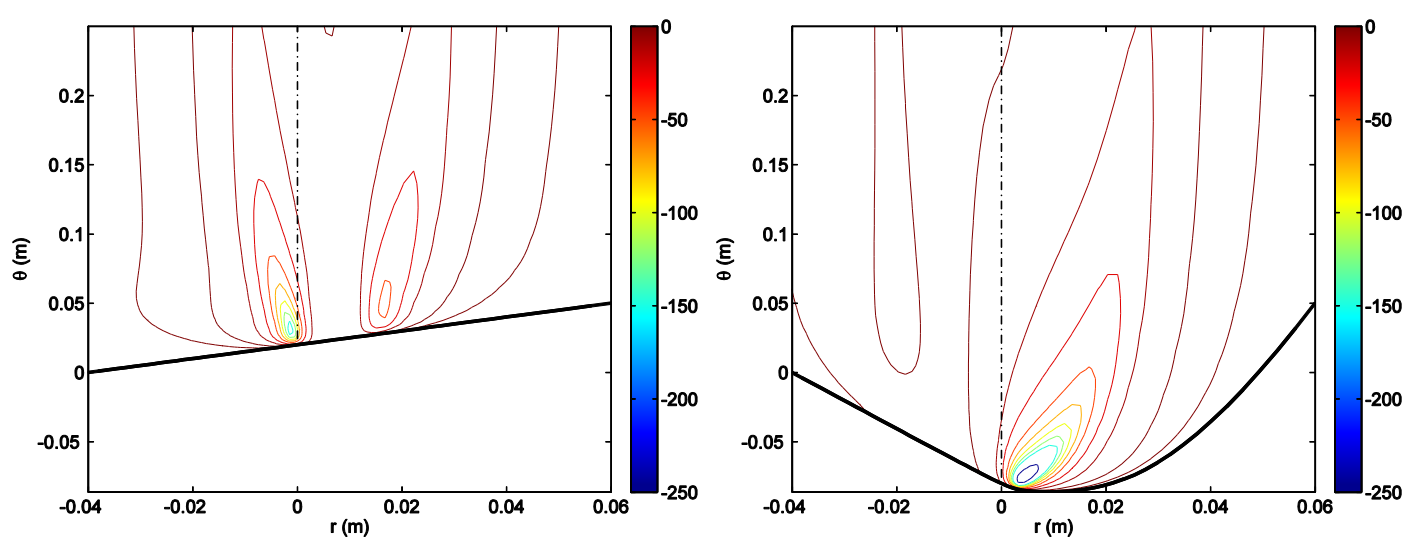

Fig. 5. Impurity radiation energy losses in initial (left) and optimized (right) configurations, ( $\left.\mathrm{MW} \mathrm{m}^{-3}\right)$.

It is an interesting and somewhat surprising feature of the optimized configuration that the peak of the power profile has shifted to the separatrix as compared to the initial straight target configuration. This can be attributed to the large shift in the energy sink due to impurity radiation. In the initial configuration, this peak was located just inside the separatrix, i.e. on the private flux side, leading to low temperature there. Due to the shift in the radiation pattern in the optimized configuration, the temperature in the private flux region has increased compared to the initial configuration, while the flux tubes in the SOL just outside the separatrix strike point are now detaching, and have lower temperatures than the surrounding flux tubes at the separatrix and just inside the private flux region. It remains to be verified whether this result can be reproduced with a more sophisticated edge code as B2-Eirene, or whether these features are very sensitive to the assumptions made in the present simple model for impurity radiation.

\section{Conclusion}

In this paper we have discussed some distinct features of divertor design problems, and motivated the use of a shape optimization framework to formulate these problems. Applying advanced adjoint based optimization algorithms, in particular a one-shot method, we are able to solve divertor design problems very efficiently. This is illustrated for an ITER-like design case, where the aim is to spread the target heat load as much as possible. 
The results above indicate that this optimal design strategy, if applied to the simplified physical model we use, automatically and rapidly identifies (re-invents) the V-shaped target concept, which in fusion research also has emerged over the past decade by experimental and extensive computational effort. This can be taken as strong indication that, indeed, this novel concept if further elaborated towards the full physical plasma boundary model, can evolve towards a new and powerful computational divertor design tool, e.g. for the first DEMO fusion power plant. Via its intrinsic sensitivity analysis, this method in principle also allows to assess the robustness of a certain design against model uncertainties, including uncertainties in magnetic equilibrium (e.g. separatrix position), impurity model,... This robustness analysis will be pursued in future work.

\section{References}

1. D. Reiter, M. Baelmans, P. Börner, Fusion Sci. Tech. 47, 172-186 (2005)

2. A. S. Kukushkin, H. D. Pacher, V. Kotov, G. W. Pacher, D. Reiter, Fusion Eng. Des. 86, $2865-$ $2873(2011)$

3. A. Jameson, L. Martinelli, N. A. Pierce, Theor. Comput. Fluid Dynamics 10, 213-237 (1998)

4. W. K. Anderson, V. Venkatakrishnan, Comput. Fluids 28, 443-480 (1999)

5. D. I. Papadimitriou, K. C. Giannakoglou, Comput. Fluids 36, No. 2, 325-341 (2007)

6. G. Kuruvila, S. Ta'asan, M. Salas, Proceedings of the 33rd Aerospace Sciences Meeting and Exhibit, AIAA, Reno, NV, U.S.A, 95-0478 (1995)

7. S. B. Hazra, V. Schulz, J. Brezillon, N. R. Gauger, J. Comput. Phys. 204, 46-64 (2005)

8. W. Dekeyser, D. Reiter, M. Baelmans, accepted for publication in Contrib. Plasma Phys. (2011)

9. S. I. Braginskii, Rev. Plasma Phys. 1, 250-311 (1965)

10. R. J. Goldston, P. H. Rutherford, Introduction to Plasma Physics (IOP Publishing, Bristol and Philadelphia, 1995)

11. V. A. Rozhansky, S. P. Voskoboynikov, E. G. Kaveeva, D. P. Coster, R. Schneider, Nucl. Fusion 41, 387-401 (2001)

12. Neuhauser et al., Plasma Phys. Control. Fusion 31, 1551 (1989) 